\title{
Enhancement of Flux Pinning in the Superconductor-Ferromagnet Bilayer $\mathrm{Nb}(\mathrm{Co} / \mathrm{Pd})$
}

\author{
Y. SyryanyY ${ }^{a}$, L.Y. Zhu ${ }^{b}$, M.Z. CiePlak ${ }^{a, b}$ AND C.L. ChIEN ${ }^{b}$ \\ ${ }^{a}$ Institute of Physics, Polish Academy of Sciences, al. Lotników 32/46, 02-668 Warsaw, Poland \\ ${ }^{b}$ Department of Physics and Astronomy, Johns Hopkins University, Baltimore, MD 21218, USA \\ The magnetometry and the magnetic force microscopy are used to study the influence of the magnetic domain \\ size on the flux pinning in a superconducting/ferromagnetic bilayer (SFB), in which the S layer is niobium and \\ the $\mathrm{F}$ layer is a $\mathrm{Co} / \mathrm{Pd}$ multilayer with perpendicular magnetic anisotropy. The domain size is pre-defined using \\ the angle-dependent demagnetization. The enhancement of pinning is found to be the strongest, up to a factor \\ of 6 , for narrow domains and small magnetic fields. This result differs from the behavior observed in the SFB \\ in which the $\mathrm{F}$ layer is $\mathrm{Co} / \mathrm{Pt}$. The difference may be attributed to the degree of the disorder in the domain pattern.
}

PACS numbers: 74.78.-w, 74.78.Fk, 74.25.Ha, 75.70.Kw, 75.70.-i, 75.70.Cn, 75.78.Fg

\section{Introduction}

The mechanisms of vortex pinning in type-II superconductors are a focus of intensive studies because of great scientific and technological interest. One of the mechanisms studied recently is the magnetic vortex pinning in the superconductor/ferromagnet bilayer (SFB), in which the ferromagnetic domains in the F-layer serve as pinning centers, interacting with the magnetic field of the vortex via the long-range electromagnetic interaction. The effect is easier to evaluate if a thin insulating buffer layer is inserted between the $\mathrm{S}$ and the F-layers, because the buffer cuts off proximity effect. The magnetic pinning is expected to act on distances comparable to the magnetic penetration depth, so it becomes important at high temperatures, when the pinning on the structural defects (acting on the distances comparable to the coherence length) is ineffective [1]. Theoretical considerations and experiments show that the magnetic domains create a pinning potential for vortices, $U=-\boldsymbol{h}_{\mathrm{v}} \cdot \boldsymbol{m}$, which is asymmetric with respect to the alignment between $\boldsymbol{h}_{\mathrm{v}}$ and $\boldsymbol{m}\left(\boldsymbol{h}_{\mathrm{v}}\right.$ is the field of the vortex, and $\boldsymbol{m}$ is the magnetic moment of the domain) [2-5].

We have evaluated recently in detail the magnetic vortex pinning in the SFB's in which the $\mathrm{S}$ layer is niobium, and $\mathrm{Co} / \mathrm{Pt}$ multilayer with perpendicular magnetic anisotropy (PMA) serves as a ferromagnet [6]. A number of interesting features have been found, including the strong dependence of the pinning enhancement on the magnetic domain pattern, and domain-induced modifications of the vortex dynamics. In the present work we extend our studies to the SFB's in which the F-layer is $\mathrm{Co} / \mathrm{Pd}$ multilayer with PMA. The domain pattern in this case is somewhat different than in the case of $\mathrm{Co} / \mathrm{Pt}$ multilayer, and we evaluate how this influences the magnetic pinning.

\section{Experimental details and results}

The two SFB's, denoted as Pd-SFB and Pt-SFB, are grown at room temperature by sputtering on $\mathrm{Si}(100)$ substrate. Schematic drawing of the SFB's is shown in Fig. 1b. The sequences of layers are $\mathrm{Pd}(10) /[\mathrm{Co}(0.5) / \mathrm{Pd}(1.5)] \times 8 / \mathrm{Si}(10) / \mathrm{Nb}(76) / \mathrm{Si}(10)$ and $\mathrm{Pt}(10) /[\mathrm{Co}(0.6) / \mathrm{Pt}(0.3)] \times 8 / \mathrm{Si}(10) / \mathrm{Nb}(76)$, for $\mathrm{Pd}-\mathrm{SFB}$ and Pt-SFB, respectively, with thickness denoted in nanometers. The coercive fields, $H_{\mathrm{c}}$, are $1500 \mathrm{Oe}$ in Pd-SFB and 775 Oe in Pt-SFB, and both samples display in the normal state square-like hysteresis loop characteristic for the PMA. The superconducting transition temperature, $T_{\mathrm{c}}$, is $8.4 \mathrm{~K}$ and $8.33 \mathrm{~K}$ in Pd-SFB and Pt-SFB, respectively. To assure similar effect of the temperature on the enhancement of pinning in both cases, the studies of pinning have been done at the same reduced temperature, $T / T_{\mathrm{c}} \approx 0.92$.

To define the domain patterns in our SFB's we demagnetize the F-layer at room temperature in the ac magnetic field tilted at an angle $\theta$ to the sample plane, as depicted in Fig. 1a. Four examples of magnetic force microscopy (MFM) images, taken for Pd-SFB demagnetized at various angles, are shown in Fig. 1c. It is seen that the demagnetization produces domain patterns with the domain width which increases with the increase of the angle $\theta$. Similar behavior have been observed by us before in the study of Pt-SFB, in which we created tunable superconducting phase diagram [7]. The effect results from the fact that the amount of domain walls defined during the demagnetization of the PMA ferromagnet is roughly proportional to the in-plane component of the ac demagnetizing field, because the magnetization is directed in-plane in the area of the domain walls.

From the MFM images we calculate the average domain width, $L$, and the standard deviation, which are shown versus $\theta$ in Fig. 2 for Pd-SFB and Pt-SFB. It is seen that in both cases the smallest width, $L \approx 0.4 \mu \mathrm{m}$, is obtained for $\theta=0$. $L$ increases quickly with increasing $\theta$ in the small $\theta$-range, and saturates for $\theta \gtrsim 15$ for Pt-SFB, and for $\theta \gtrsim 25$ for Pd-SFB. The total increase of $L$ is by a factor of 1.7 and 3.8 for $\mathrm{Pt}$ and $\mathrm{Pd}$, respectively. A factor of two larger increase of $L$ in case of $\mathrm{Pd}-\mathrm{SFB}$ may be related to a factor of two larger $H_{\mathrm{c}}$. 
(a)

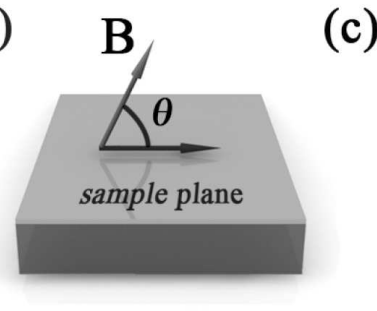

(b)

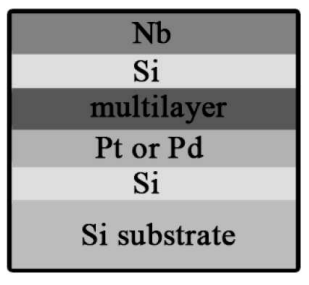

(c)

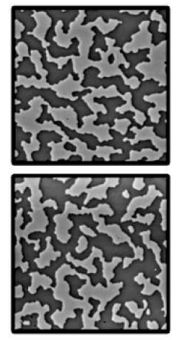

$90^{\circ}$

$45^{0}$

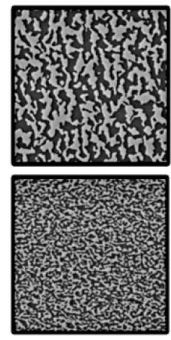

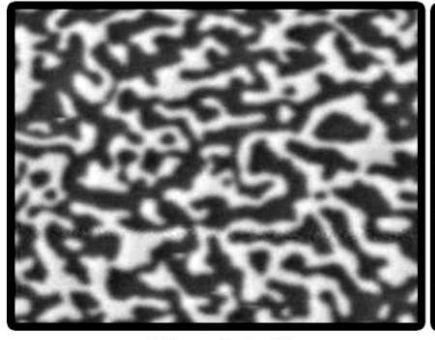

$\mathrm{Co} / \mathrm{Pd}$

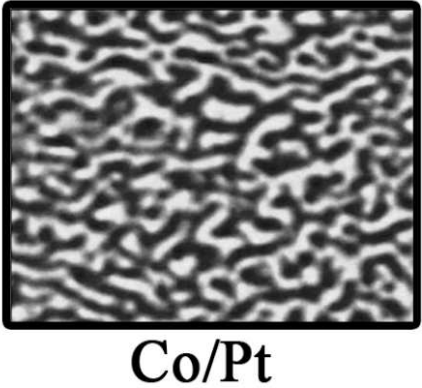

$\mathrm{Co} / \mathrm{Pt}$

Fig. 3. The MFM images for Pd-SFB and Pt-SFB demagnetized at $\theta=0^{\circ}$. The image sizes are $7 \times 9 \mu \mathrm{m}$.

loops of magnetization for the $\mathrm{Pd}-\mathrm{SFB}$ with pre-defined domain patterns, measured with the SQUID magnetometer at $T<T_{\mathrm{c}}$. The measurement is restricted to $H \ll H_{\mathrm{c}}$, so it has no effect on the domain pattern (the magnetization of the $\mathrm{Pd}$-layer, measured just above the $T_{\mathrm{c}}$, is subtracted from the data). The most inside curve shows the hysteresis loop for the saturated F-layer, when the magnetic pinning is negligible. The peak-to-peak width of the hysteresis, $\Delta M_{\mathrm{pp}}$, is seen to be the smallest in this case. All the data measured for demagnetized SFB show larger $\Delta M_{\mathrm{pp}}$, indicating the enhancement of pinning by the magnetic domains. $\Delta M_{\mathrm{pp}}$ is the largest for $\theta=0$, and gradually decreases as $\theta$ grows.

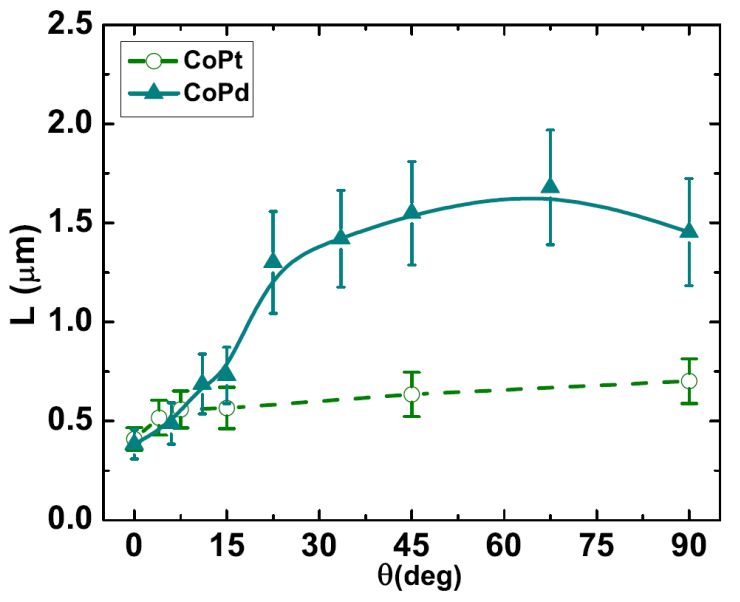

Fig. 2. Dependence of the average domain width $L$ on the angle $\theta$ for the SFB's with $\mathrm{Co} / \mathrm{Pd}$ and $\mathrm{Co} / \mathrm{Pt}$ multilayers. The error bars show the standard deviation.

Let us note that the standard deviation is also a factor of about two larger in case of $\mathrm{Pd}-\mathrm{SFB}$, it amounts to about $37 \%$, while it is only about $16 \%$ in case of Pt-SFB. This is presumably related to the larger thickness of the nonmagnetic $(\mathrm{Pd})$ spacer in the $\mathrm{Co} / \mathrm{Pd}$ multilayer. It contributes to the creation of larger amount of structural defects which pin the domain walls, making them more disordered. In Fig. 3 we compare the images of two SFB's, both demagnetized at $\theta=0$. The larger disorder in the $\mathrm{Pd}-\mathrm{SFB}$ is clearly evident.

We now turn the attention to the effect of pinning in the superconducting state. Figure 4 shows hysteresis

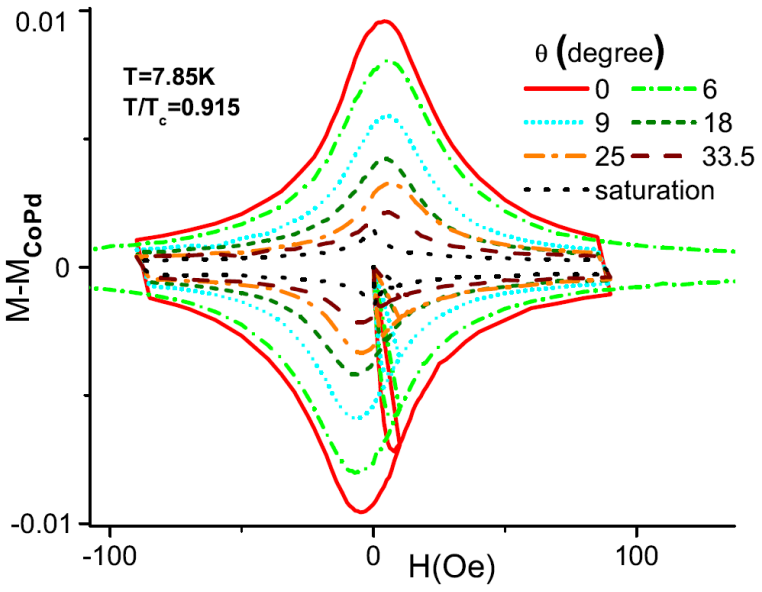

Fig. 4. The hysteresis loops for Pd-SFB, demagnetized at various angles $\theta$, and for saturated F-layer, measured at $T=7.85 \mathrm{~K}$.

To quantify this effect we define the enhancement of pinning as the ratio $W=\Delta M(\theta) / \Delta M_{\text {sat }}$, where $\Delta M(\theta)$ is measured for any $\theta$, and $\Delta M_{\text {sat }}$ is measured for saturated F-layer. In addition to the peak-to-peak width $\Delta M_{\mathrm{pp}}$, we also extract the width of the hysteresis loop at $H=85$ Oe, $\Delta M_{85}=M_{+}(H=85 \mathrm{Oe})-M_{-}(H=85 \mathrm{Oe})$, where $M_{+}\left(M_{-}\right)$is the positive (negative) branch of the magnetization. The dependence of $W$ on $\theta$ for $\mathrm{Pd}-\mathrm{SFB}$ and Pt-SFB is shown in Fig. 5, both for $H=85 \mathrm{Oe}$, and for the peak-to-peak width, i.e. for low- $H$ range. 
We see that the pinning enhancement is larger for Pt-SFB, both for the low $H$ and for 85 Oe. However, the values of $W$ measured at low fields and at low $\theta$ are quite close to each other in the two SFB's, about 6 in Pd-SFB, and about 7.4 in Pt-SFB. The comparable enhancement of pinning at low $H$ has a straightforward explanation: when the vortex density is very low, all vortices are pinned very efficiently by narrow domains in both SFB's. Slightly smaller $W$ (by about 20\%) in the $\mathrm{Pd}-\mathrm{SFB}$ is most likely related to the more disordered domain pattern in the $\mathrm{Pd}-\mathrm{SFB}$, which contains smaller amount of narrow domains.

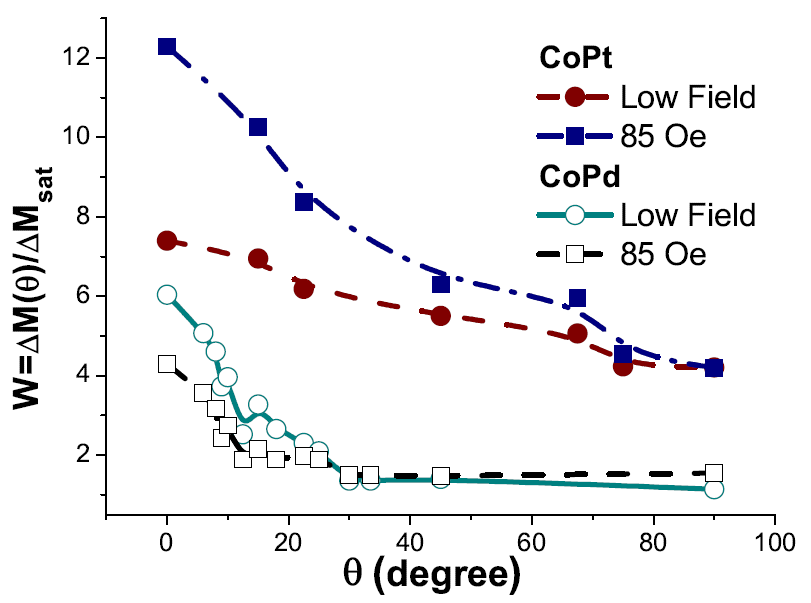

Fig. 5. Enhancement of pinning by the angle-dependent demagnetization for CoPt and CoPd samples, for low fields (circles), and for $H=85$ Oe (squares).

With increasing $\theta$ we see a suppression of $W$ measured at low $H$ in both SFB's. The faster decrease of $W$ in the $\mathrm{Pd}-\mathrm{SFB}$ is partly accounted for by the faster increase of the domain size, but this is not the only factor. To see this we extract the values of $W$ for the two SFB's at the same $L=0.7 \mu \mathrm{m}$ (which is the largest domain width in Pt-SFB). We get $W=4.2$ in Pt-SFB, and $W=2.54$ in Pd-SFB. Therefore, with increasing $\theta$ the $W$ is suppressed somewhat more strongly in Pd-SFB. The suppression of $W$ is caused by the fact that the wider domains trap vortices less efficiently, providing instead channels for easy flow of vortices. The larger standard deviation in the Pd-SFB contributes to stronger suppression of $W$.

Very different behavior of $W$ is observed in the two SFB's at 85 Oe. In Pd-SFB the $W$ measured at 85 Oe is reduced in comparison with the low- $H$ value, while in $\mathrm{Pt}-\mathrm{SFB}$ it is enhanced. The largest differences between high- $H$ and low- $H$ values are seen for small $\theta$, with increasing $\theta$ the curves for high- $H$ and low- $H$ approach each other. The most striking is the large $W$ observed in Pt-SFB. It reaches about 12, which is a record-high enhancement of pinning, never reported before.
To explain this behavior we note first that we have recently succeeded in extracting, from the transport measurements, the activation energy $U$ for the vortex pinning in the Pt-SFB [7]. The results indicate that the $U$ has maxima in the vicinity of several matching fields, in particular, it is enhanced at $H \approx 90$ Oe. The matching effects appear as a result of pinning of the single-vortex or double-vortex chains inside long, narrow domains. The SFB examined in the transport experiment has $L$ somewhat smaller than the present SFB, so we cannot use these results for quantitative comparison. Nevertheless, qualitatively it is clear that the matching effects are responsible for large $W$ observed at 85 Oe in Pt-SFB.

The suppression of $W$ at 85 Oe in case of $\mathrm{Pd}-\mathrm{SFB}$ is most likely caused by the disorder in the domain pattern. The disorder disrupts matching, so that smaller flux density is pinned by smaller amount of narrow domains, and flux flows easier in the vicinity of large domains. The transport measurements for Pd-SFB are planned to verify this conclusion.

\section{Conclusions}

The enhancement of pinning, extracted from the magnetization measurements, has been compared for two SFB's, built with the same superconducting $\mathrm{Nb}$ layer, but two different ferromagnetic layers, $\mathrm{Co} / \mathrm{Pd}$ and $\mathrm{Co} / \mathrm{Pt}$ multilayers with PMA. The domain patterns have been predefined using angle-dependent demagnetization procedure, which allows to tune domain width. It is found that the pinning enhancement is weaker in $\mathrm{Co} / \mathrm{Pd}$, which we propose to explain by more disordered domain pattern.

\section{Acknowledgments}

This work was supported by Polish MNiSW grant N202 058 32/1202, and by NSF grant DMR05-20491.

\section{References}

[1] L.N. Bulaevskii, E.M. Chudnovsky, M.P. Maley, Appl. Phys. Lett. 76, 2594 (2000).

[2] M.V. Milosevic, S.V. Yampolskii, F.M. Peeters, Phys. Rev. B 66, 174519 (2002); I.F. Lyuksyutov, V.L. Pokrovsky, Adv. Phys. 54, 67 (2004).

[3] M. Lange, M.J. Van Bael, V.V. Moshchalkov, Y. Bruynseraede, Appl. Phys. Lett. 81, 322 (2002).

[4] M.Z. Cieplak, X.M. Cheng, C.L. Chien, Hai Sang, J. Appl. Phys. 97, 026105 (2005).

[5] M.Z. Cieplak, Z. Adamus, A. Abal'oshev, I. Abal'osheva, M. Berkowski, X.M. Cheng, Hai Sang, C.L. Chien, Phys. Status Solidi C 2, 1650 (2005).

[6] Z. Adamus, M.Z. Cieplak, A. Abal'oshev, M. Kończykowski, X.M. Cheng, L.Y. Zhu, C.L. Chien, Acta Phys. Pol. A 111, 95 (2007); M.Z. Cieplak, Z. Adamus, M. Kończykowski, L.Y. Zhu, C.L. Chien, ibid., 114, 23 (2008); M.Z. Cieplak, Z. Adamus, M. Kończykowski, L.Y. Zhu, X.M. Cheng, C.L. Chien, to be published.

[7] L.Y. Zhu, M.Z. Cieplak, C.L. Chien, to be published. 